\title{
Explicit Solution to a Robust Queueing Control Problem*
}

\author{
Paul Dupuis \\ Lefschetz Center for Dynamical Systems \\ Brown University \\ Providence, R.I. 02912 \\ USA
}

\begin{abstract}
We consider the robust optimal control of a law of large numbers approximation of a stochastic network. The robust control problem is formulated as a differential game, with one player choosing the policies that determine service and routing assignments, and the other choosing quantities such as the arrival and service rates, subject to constraints. The cost to be minimized by the first player and maximized by the second is the time till the origin is reached. An explicit formula is given for the value function, and some of its basic properties are studied.
\end{abstract}

\section{Introduction}

This paper considers the problem of robust service and routing control for a network of servers. Consider such a network, and assume that at each station there are a finite number of distinct customer classes, each with its own buffer. In this paper we will work directly with what is sometimes called a "fluid" model for the network [16]. Models of this sort are usually obtained as law of large numbers approximations to more detailed models [5, 15], and are particularly appealing because in many cases related optimization problems admit closed form solutions [20, 21, 11].

Another feature of the networks we consider is model uncertainty, such as uncertainty in the arrival and service rates. To deal with model uncertainty we adapt the differential game formulation of robust control for

${ }^{*}$ This research was supported in part by the National Science Foundation (NSF-DMS0072004, NSF-ECS-9979250) and the Army Research Office (DAAD19-99-1-0223). 


\section{Report Documentation Page}

Form Approved

OMB No. 0704-0188

Public reporting burden for the collection of information is estimated to average 1 hour per response, including the time for reviewing instructions, searching existing data sources, gathering and maintaining the data needed, and completing and reviewing the collection of information. Send comments regarding this burden estimate or any other aspect of this collection of information,

including suggestions for reducing this burden, to Washington Headquarters Services, Directorate for Information Operations and Reports, 1215 Jefferson Davis Highway, Suite 1204, Arlington

VA 22202-4302. Respondents should be aware that notwithstanding any other provision of law, no person shall be subject to a penalty for failing to comply with a collection of information if it

does not display a currently valid OMB control number.

1. REPORT DATE

2001

4. TITLE AND SUBTITLE

Explicit Solution to a Robust Queueing Control Problem

6. AUTHOR(S)

7. PERFORMING ORGANIZATION NAME(S) AND ADDRESS(ES)

Brown University,Division of Applied Mathematics,182 George Street,Providence,RI,02912

9. SPONSORING/MONITORING AGENCY NAME(S) AND ADDRESS(ES)

3. DATES COVERED

00-00-2001 to 00-00-2001

5a. CONTRACT NUMBER

5b. GRANT NUMBER

5c. PROGRAM ELEMENT NUMBER

5d. PROJECT NUMBER

5e. TASK NUMBER

5f. WORK UNIT NUMBER

8. PERFORMING ORGANIZATION

REPORT NUMBER

10. SPONSOR/MONITOR'S ACRONYM(S)

11. SPONSOR/MONITOR'S REPORT

$\operatorname{NUMBER}(S)$

12. DISTRIBUTION/AVAILABILITY STATEMENT

Approved for public release; distribution unlimited

13. SUPPLEMENTARY NOTES

14. ABSTRACT

15. SUBJECT TERMS

16. SECURITY CLASSIFICATION OF:

a. REPORT

b. ABSTRACT

unclassified

unclassified

c. THIS PAGE

17. LIMITATION OF ABSTRACT

18. NUMBER

OF PAGES

29 19a. NAME OF

RESPONSIBLE PERSON

\section{unclassified}


unconstrained nonlinear systems [14]. Thus we consider a network where there are two players. One player in the game will represent the "true" control (e.g., service assignments and routing decisions). The other player represents the uncertain or poorly modeled aspects of the system (e.g., arrival and service rates). In keeping with existing convention, we will refer to this latter control as "nature." The two players are antagonistic, with the first player attempting to maintain good system performance.

Differential game formulations provide a powerful tool for the design of robust controls $[3,14]$. In many situations knowledge of the true system is limited. System parameters (e.g., arrival rates) may drift with time, and statistical properties (e.g., correlations) may also be unstable. There may be aspects of the system that are left unmodeled, either because they cannot be estimated in any reliable way, or because they lead to a model that is too complicated to be useful. This is a common occurence in stochastic networks, where the network to be controlled is often a sub-network of some larger system, and "full state information" is simply not available to the controller of the sub-network.

In situations like these the use of a single "nominal" model can be problematic. For example, just as in the case of unconstrained systems one can construct examples where controls that are optimal in some sense for the nominal model perform poorly when the model is perturbed even slightly. A differential game formulation allows one to contruct controls that perform uniformly well over a class of perturbations of the nominal model, with each choice of nature's control corresponding to a different perturbation of the design model. It is, of course, this insensitivity to model perturbations that warrants the term "robust" control. Variations of different kinds can be accomodated through the choice of the cost structure, and one can carefully balance the pursuit of optimality with respect to a nominal model against the need to provide good performance for a range of models. The main result of this paper is the explicit solution to a robust control problem for a network. By explicit what we mean is that the value function can be represented in terms of a finite dimensional optimization problem, and that from this value function one can obtain controls with specific robust properties.

In formulating the differential game special attention must be paid to the cost applied to nature's control, since this determines the degree to which model perturbations are allowed. Within the realm of "fluid" models there are at least two types of cost structures that are natural. One is a cost that simply imposes a constraint on the model parameters. We will refer to this as the case of a "hard constraint." An alternative is to make nature pay an increasing cost for perturbations away from the nominal model, and 
we will refer to this type of cost as corresponding to "soft constraints." Hard constraints turn out to be mathematically simpler for fluid models of networks, even though the reverse seems to be true for unconstrained systems. In the present paper we will focus on the case of hard constraints, and defer the case of soft constraints to future work.

Besides the cost that nature must pay, one must also specify the cost that the true control faces. In this paper the cost we consider is the time to move the state of the system from an arbitrary position to zero (i.e., all queues empty). The true control will try to minimize this time, while the opposing control will attempt to delay it as much as possible. This cost seems to be a natural analogue, in the setting of constrained systems, of the familiar quadratic cost for unconstrained systems. In particular, it leads to controls with optimal robust stability (in addition to optimal robust performance), and it also allows for a fairly explicit closed form solution.

An outline of the paper is as follows. In Section 2 we give a precise formulation of the game problem, and show through examples how various systems can be put into this framework. Sections 3 includes the main result of the paper, which is a finite dimensional $\mathrm{max} / \mathrm{min}$ representation for the value function of the game introduced in Section 2. Qualitative properties of the value function (convexity, differentiability, etc.) are also discussed in Section 3. The proof of this representation is given in Section 4. The concluding Section 5 formally discusses how an optimal true control can be constructed in feedback form. A proof of the existence of value for the games we consider is given in an Appendix.

\section{Formulation of the Control Problem}

In this section we formulate the robust control problem as a constrained deterministic differential game. As discussed in the introduction, the model we use can be viewed as a law of large numbers approximation to a more detailed stochastic model. This connection will be used for interpretive purposes throughout the section. The state space of the process is $\mathbb{R}_{+}^{N}$, and one can interpret each of the components as a queue length associated with a specific customer class.

The formulation of the model involves two collections of $N$-dimensional vectors. The first are the directions of constraint, which we designate by $\left\{d_{i}, i=1, \ldots, N\right\}$. These vectors are used to define the Skorokhod or reflection map, which properly corrects the dynamics of the model when one or more components of the state are zero (i.e., one or more customer classes are 
empty). The second collection is designated $\left\{v_{j k}, j=1, \ldots, J, k=1, \ldots, K\right\}$, and is used to define the dynamics of the system away from the boundary. Nature's control takes values in a compact convex set $\mathcal{A} \subset \mathbb{R}^{K}$, and the in$\operatorname{dex} j \in\{1, \ldots, J\}$ corresponds to one of the possible "pure" service/routing configurations the true controller can select (illustrative examples will be given below). If nature chooses the control $\alpha \in \mathcal{A}$ and the true control is the pure configuration $j$, then the quantity $\sum_{k=1}^{K} \alpha_{k} v_{j k}$ characterizes the (law of large numbers) evolution of the network when the state of the network is away from $\partial \mathbb{R}_{+}^{N}$. More general service/routing policies can be obtained by considering convex combinations of the pure controls, in which case the velocity of the system is given by

$$
F(\rho, \alpha) \doteq \sum_{j=1}^{J} \sum_{k=1}^{K} \rho_{j} \alpha_{k} v_{j k}
$$

where

$$
\rho=\left(\rho_{1}, \ldots, \rho_{J}\right) \in \mathcal{S} \doteq\left\{x \in \mathbb{R}^{J}: x_{j} \geq 0, j=1, \ldots, J, \sum_{j=1}^{J} x_{j}=1\right\},
$$

and $\rho_{j}$ is the fraction of time allocated to the pure configuration $j$.

We will assume the following condition on the directions of constraint. The condition is by now classical in the study of approximations to queueing networks, and is called the Harrison-Reiman condition in [10]. It was first used in [13]. Although the Harrison-Reiman condition is usually associated with single class networks, it also defines the proper Skorokhod Problem for many formulations of controlled multiclass networks as well. Note that the condition is the original Harrison-Reiman condition, and not the generalization that is also studied in [10].

Condition 2.1 For each $i \in\{1, \ldots, N\}$

$$
\left(d_{i}\right)_{i}=1 \text {, and }\left(d_{i}\right)_{j} \leq 0 \text { for } j \neq i .
$$

Let $D$ be the matrix whose ith column is $d_{i}$. Then the spectral radius of $I-D$ is less than 1.

The following simple examples illustrate the role these different quantities play. The $i$ th unit basis vector is denoted by $e_{i}$. Some of the most difficult aspects in the control of networks are due to feedback and the interactions between different servers. ¿From this perspective, the first two 
examples are too simple to be of great interest. Also, it should be noted that the game formulation we consider in this paper only allows routing at the "fringes" of the network, and not between nodes. We hope to consider the more general routing problem in future work.

Example 1. The first example is a simple routing control problem. The rate of arrivals to the router is $\lambda(t)$, and the service rates of the two servers are $\mu_{1}(t)$ and $\mu_{2}(t)$, respectively. The system is illustrated in Figure 1.

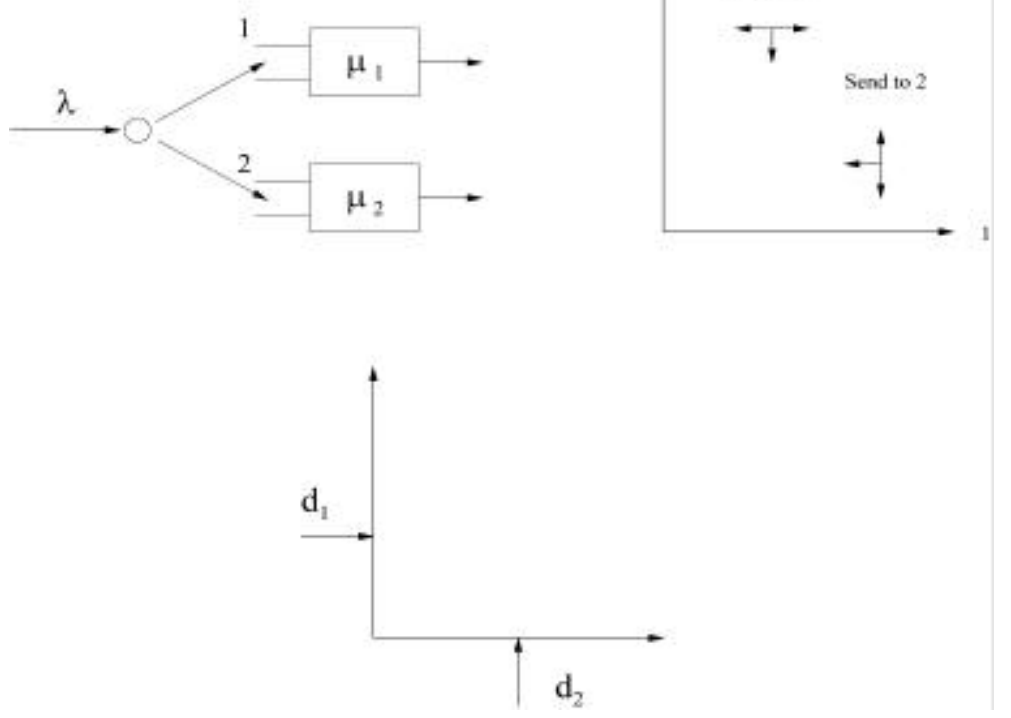

Figure 1: Simple routing control

This model is put into the framework described above by setting

$$
\begin{array}{lll}
\alpha_{1}=\mu_{1} & v_{1,1}=-e_{1} & v_{2,1}=-e_{1} \\
\alpha_{2}=\mu_{2} & v_{1,2}=-e_{2} & v_{2,2}=-e_{2} \\
\alpha_{3}=\lambda & v_{1,3}=e_{1} & v_{2,3}=e_{2}
\end{array} .
$$

The choice of $\mathcal{A}$ determines the uncertainties and perturbations against which the optimal true control will be robust. For example, if the nominal service and arrival rates are $\bar{\lambda}=1$ and $\bar{\mu}_{i}=1, i=1,2$, and if the service rates are well modeled and the arrival rate less so, then one might consider a set of the form

$$
\mathcal{A}=\left[\bar{\mu}_{1}^{L}, \bar{\mu}_{1}^{U}\right] \times\left[\bar{\mu}_{2}^{L}, \bar{\mu}_{2}^{U}\right] \times\left[\bar{\lambda}^{L}, \bar{\lambda}^{U}\right]=[.9,1.1] \times[.9,1.1] \times[.5,1.5] .
$$


This model is very simple, and perhaps too simple to capture any "probabilistic" intuition. For example, there is no constraint on combinations of $\mu_{1}$ and $\mu_{2}$. ¿From a probabilistic perspective one might imagine that it is less likely that both of these parameters would equal their minimum value at the same time. The introduction of a constraint to account for this would lead to a set $\mathcal{A}$ with a "curved" boundary.

One might also wish to consider an increasing family of sets $\mathcal{A}(c)$ indexed by $c \in[0, \infty)$, and with $\mathcal{A}(0)$ just the nominal model. The largest $c$ such that a certain robust performance measure can be met (e.g., finiteness of the value function) is an important quantity. In particular, it characterizes the control that is most robust, where the sense of robustness is determined by the shape of $\mathcal{A}$ and the relative uncertainty it assigns to different aspects of the network.

We return our consideration to the particular example of Figure 1. If a service is attempted at server 1 and the queue is empty then the proper compensating action is simply to return queue 1 to the level zero. As a consequence, the direction of constraint for the corresponding face is just $d_{1}=(1,0)$. A corresponding remark applies to queue 2 .

Example 2. Here we consider a simple service control problem. The system is illustrated in Figure 2.

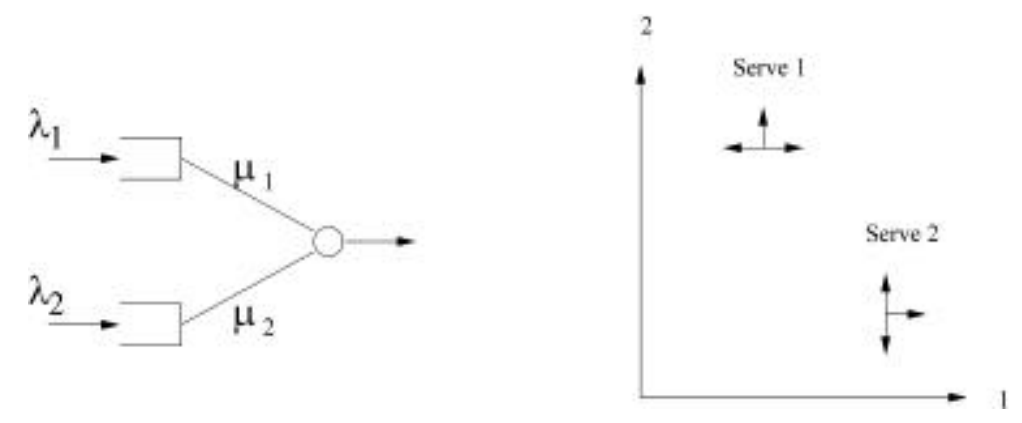

Figure 2: Simple service control

The Skorokhod Problem for this is the same as that for Example 1. This 
model is put into the standard framework by setting

$$
\begin{array}{lll}
\alpha_{1}=\mu_{1} & v_{1,1}=-e_{1} & v_{2,1}=0 \\
\alpha_{2}=\mu_{2} & v_{1,2}=0 & v_{2,2}=-e_{2} \\
\alpha_{3}=\lambda_{1} & v_{1,4}=e_{1} & v_{2,4}=e_{1} \\
\alpha_{4}=\lambda_{2} & v_{1,4}=e_{2} & v_{2,4}=e_{2}
\end{array}
$$

Example 3. This example considers a network of servers, and as a consequence the associated Skorokhod Map is more involved. The network is illustrated in Figure 3. Since there are 6 customer classes the domain is $\mathbb{R}_{+}^{6}$. Suppose the service rate for class $i$ is $\mu_{i}(t)$ and the arrival rate is $\lambda(t)$.

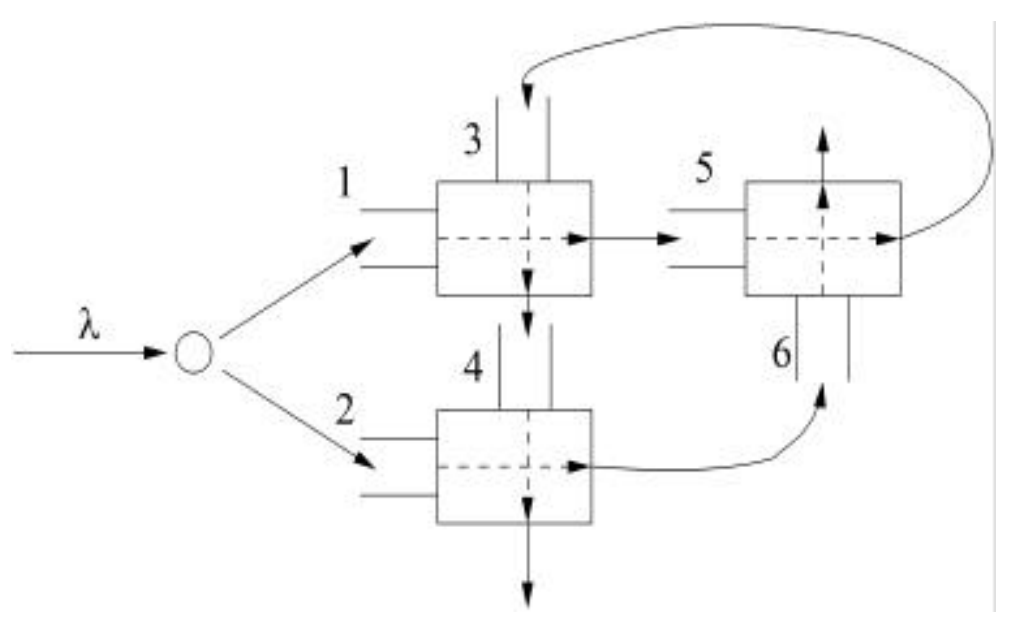

Figure 3: Network model

An example of a pure configuration (labeled say $j$ ), is to route to class 1 and serve classes 3,2 and 5 . If we let $\left(\alpha_{1}, \ldots, \alpha_{6}, \alpha_{7}\right)=\left(\mu_{1}, \ldots, \mu_{6}, \lambda\right)$, then the vectors $v_{j k}$ for $k=2,3,5,7$ are

$$
v_{j 2}=\left(-e_{2}+e_{6}\right), v_{j 3}=\left(-e_{3}+e_{4}\right), v_{j 5}=\left(-e_{5}+e_{3}\right), v_{j 7}=e_{1},
$$

while $v_{j 1}=v_{j 4}=v_{j 6}=0$. The velocity of the network under this configuration is $\mu_{2} v_{j 2}+\mu_{3} v_{j 3}+\mu_{5} v_{j 5}+\lambda v_{j 7}$.

If a service is attempted for say customer class $i=3$ and the queue is empty, then queue 3 must be returned to zero and in addition queue 4 must be reduced by the same amount. Consequently, the proper direction 
of constraint for face $i=3$ is $d_{3}=\left(e_{3}-e_{4}\right)$. Analogous considerations can be used to identify all other directions of constraint.

Example 4. In some problems there is randomized (uncontrolled) routing. For example, after service a fraction $\theta_{j}$ of the class $i$ customers may become class $j$ customers, and a fraction $\theta_{0}=1-\sum_{j=1, j \neq i}^{J} \theta_{j}$ of the customers could leave the system. Let $\left(d_{i}\right)_{j}=-\theta_{j}$ if $j \neq i$ and $\left(d_{i}\right)_{i}=1$. Then the direction of constraint is $d_{i}$ on the face $\left\{x \in \mathbb{R}_{+}^{N}: x_{i}=0\right\}$, and the reason is the same as in the last case: compensating for a "fictitous" service of a customer of class $i$ requires a boost to coordinate $i$ and a corresponding decrease in coordinate $j$ with constant of proportionality $\theta_{j}[18]$.

To formulate the robust control problem we must specify the dynamics. Let

$$
C_{+}\left([0, \infty): \mathbb{R}^{N}\right) \doteq\left\{\psi \in C\left([0, \infty): \mathbb{R}^{N}\right): \psi(0) \in \mathbb{R}_{+}^{N}\right\},
$$

where $C\left([0, \infty): \mathbb{R}^{N}\right)$ is the usual space of continuous functions with the sup norm metric, and suppose that a set of vectors that satisfy Condition 2.1 is given. For each point $x$ on the boundary of $\mathbb{R}_{+}^{N}$ let

$$
d(x) \doteq\left\{\sum_{i \in I(x)} a_{i} d_{i}: a_{i} \geq 0,\left\|\sum_{i \in I(x)} a_{i} d_{i}\right\|=1\right\}
$$

where $I(x) \doteq\left\{i: x_{i}=0\right\}$. The Skorokhod Problem assigns to every path $\psi \in C_{+}\left([0, \infty): \mathbb{R}^{N}\right)$ a path $\phi$ that starts at $\phi(0)=\psi(0)$, but is constrained to $\mathbb{R}_{+}^{N}$ as follows. If $\phi$ is in the interior of $\mathbb{R}_{+}^{N}$ then the evolution of $\phi$ mimics that of $\psi$, in that the increments of the two functions are the same until $\phi$ hits the boundary of $\mathbb{R}_{+}^{N}$. When $\phi$ is on the boundary a constraining "force" is applied to keep $\phi$ in the domain, and this force can only be applied in one of the directions $d(\phi(t))$, and only for $t$ such that $\phi(t)$ is on the boundary. The precise definition is as follows. For $\eta \in C\left([0, \infty): \mathbb{R}^{N}\right)$ and $t \in[0, \infty)$ we let $|\eta|(t)$ denote the total variation of $\eta$ on $[0, t]$ with respect to the Euclidean norm on $\mathbb{R}^{N}$.

Definition 2.1 Let $\psi \in C_{+}\left([0, \infty): \mathbb{R}^{N}\right)$ be given. Then $(\phi, \eta)$ solves the $S P$ for $\psi$ (with respect to $\mathbb{R}_{+}^{N}$ and $\left.d_{i}, i=1, \ldots, N\right)$ if $\phi(0)=\psi(0)$, and if for all $t \in[0, \infty)$

1. $\phi(t)=\psi(t)+\eta(t)$,

2. $\phi(t) \in G$, 
3. $|\eta|(t)<\infty$,

4. $|\eta|(t)=\int_{[0, t]} 1_{\left\{\phi(s) \in \partial \mathbb{R}_{+}^{N}\right\}} d|\eta|(s)$,

5. There exists a Borel measurable function $\gamma:[0, \infty) \rightarrow \mathbb{R}_{+}^{N}$ such that $d|\eta|$-almost everywhere $\gamma(t) \in d(\phi(t))$, and such that

$$
\eta(t)=\int_{[0, t]} \gamma(s) d|\eta|(s) \text {. }
$$

Note that $\eta$ changes only when $\phi$ is on the boundary, and only in the directions $d(\phi)$.

Under Condition 2.1 the Skorokhod Problem has a solution for all $\psi \in$ $C_{+}\left([0, \infty): \mathbb{R}^{N}\right)$. In addition, the mapping $\psi \rightarrow \phi$ is Lipschitz continuous $[8,13]$.

We next define a constrained ordinary differential equation. As is proved in [8], one can define a projection $\pi: \mathbb{R}^{N} \rightarrow \mathbb{R}_{+}^{N}$ that is consistent with the constraint directions $\left\{d_{i}, i=1, \ldots, N\right\}$, in that $\pi(x)=x$ if $x \in \mathbb{R}_{+}^{N}$, and if $x \notin \mathbb{R}_{+}^{N}$ then $\pi(x)-x=\alpha r$, where $\alpha \geq 0, \pi(x) \in \partial \mathbb{R}_{+}^{N}$, and $r \in d(\pi(x))$. Figure 4 illustrates the projection for a two dimensional problem.

Figure 4: The discrete projection

With this projection given, we can define for each point $x \in \partial \mathbb{R}_{+}^{N}$ and each $v \in \mathbb{R}^{N}$ the projected velocity

$$
\pi(x, v) \doteq \lim _{\Delta \downarrow 0} \frac{\pi(x+\Delta v)-\pi(x)}{\Delta} .
$$


For details on why this limit is always well defined and further properties of the projected velocity we refer to [6, Section 3 and Lemma 3.8] and [7]. The dynamical model for the game we consider is then given by

$$
\dot{\phi}(t)=\pi(\phi(t), F(\rho(t), \alpha(t))),
$$

where

$$
F(\rho(t), \alpha(t))=\sum_{j=1}^{J} \rho_{j}(t) \alpha_{k}(t) v_{j k},
$$

and for all $t \in[0, \infty)$ the true control $\rho(t)$ takes values in the set $\mathcal{S}$ and nature's control $\alpha(t)$ takes values in the set $\mathcal{A}$. According to the Skorokhod Problem, the velocity $F(\rho, \alpha)$ governs the evolution of the network when all states are positive. When one or more states are negative, the projection of the velocity provides the proper correction to the dynamics due to nonnegativity constraints.

An absolutely continuous function $\phi:[0, \infty) \rightarrow \mathbb{R}_{+}^{N}$ is a solution to (2) if the equation is satisfied in an a.e. sense in $t$. By using the regularity properties of the associated Skorokhod Map, one can prove that all the standard qualitative properties (existence and uniqueness of solutions, stability with respect to perturbations, etc.) hold [8]. In fact, because of the particularly simple nature of the right hand side (i.e., $\pi(\phi(t), \beta(t))$ rather than $\pi(\phi(t), b(\phi(t))+\beta(t))$ for some function $b)$, one can show that $\phi$ solves (2) if and only if $\phi$ is the image of $\psi(t) \doteq \int_{0}^{t} F(\rho(s), \alpha(s)) d s+x$ under the Skorokhod Map, in which case all such issues become trivial [8].

The ODE (2) defines the dynamics for the game that we will consider. The cost we consider is the time for the state to reach the origin, which the true control will attempt to minimize and which nature will try to prolong. As usual in differential games, one must deal with the issue of which player has the "information advantage" [12]. For the problems we consider it will always turn out that the game has value, and so the value function will be the same regardless of who has the information advantage.

We use the standard Elliot-Kalton formulation of the game. Define the spaces of (open loop) controls

$$
N \doteq\{\rho:[0, \infty) \rightarrow \mathcal{S}: \rho \text { is measurable }\}
$$

and

$$
M \doteq\{\alpha:[0, \infty) \rightarrow \mathcal{A}: \alpha \text { is measurable }\} .
$$

We identify any two controls that are equal almost everywhere. Given $x \in$ $\mathbb{R}_{+}^{N}$, the dynamics of the game are given by (2) and (3). Associated with 
these dynamics is the cost

$$
C_{x}(\rho, \alpha) \doteq \tau_{x},
$$

where $\tau_{x} \doteq \inf \{t \geq 0: \phi(t)=0\}$. A mapping $\theta: N \rightarrow M$ is said to be a strategy for the maximizing player if for each $s \geq 0$ and $\rho, \hat{\rho} \in N$

$$
\rho(t)=\hat{\rho}(t) \text { for a.e. } 0 \leq t \leq s
$$

implies

$$
\theta[\rho](t)=\theta[\hat{\rho}](t) \text { for a.e. } 0 \leq t \leq s .
$$

A strategy for the minimizing player, which will be denoted by $\delta$, is defined in an analogous manner. We denote by $\Theta$ the set of all maximizing strategies and by $\Delta$ the set of all minimizing ones. The lower value of the game and the upper value of the game are defined by

$$
V^{-}(x) \doteq \inf _{\delta \in \Delta} \sup _{\alpha \in M} C_{x}(\delta[\alpha], \alpha)
$$

and

$$
V^{+}(x) \doteq \sup _{\theta \in \Theta} \inf _{\rho \in N} C_{x}(\rho, \theta[\rho]),
$$

respectively. If $V^{-}(x)=V^{+}(x)$, then the game is said to have value.

Let $V: \mathbb{R}^{N} \rightarrow \mathbb{R}$. For points $x \in \mathbb{R}^{N}$ and directions $w \in \mathbb{R}^{N}$ for which the limit exists, we let $D_{w} V(x)$ denote the directional derivative in direction $w$ at $x$ :

$$
D_{w} V(x) \doteq \lim _{a \downarrow 0} \frac{V(x+a w)-V(x)}{a} .
$$

We say that $V$ is radially linear if $V(a x)=a V(x)$ for all $x \in \mathbb{R}^{N}$ and $a \in[0, \infty)$.

\section{Representation for the Value Function}

For $V^{+}(x)$ and $V^{-}(x)$ to be finite we will need some conditions. Define the convex cone

$$
\mathcal{C} \doteq\left\{-\sum_{i=1}^{N} a_{i} d_{i}: a_{i} \geq 0, i=1, \ldots, N\right\}
$$

which is the negative of the cone of constraint directions that are allowed at the origin. As observed in [6], this cone can be used to characterize stability conditions for $(2)$. 
The following formula gives an explicit representation for the value of the game defined in the last section. The precise statement is given at the end of the section, and the proof is given in Section 4. Recall that $F(\rho, \alpha) \doteq \sum_{j=1}^{J} \sum_{k=1}^{K} \rho_{j} \alpha_{k} v_{j k}$. Then set

$$
W(x) \doteq \sup _{\alpha \in \mathcal{A}} \inf _{\rho \in \mathcal{S}} \inf \{\sigma: x+\sigma F(\rho, \alpha) \in \mathcal{C}\} .
$$

We will also make use of

$$
W_{\alpha}(x) \doteq \inf _{\rho \in \mathcal{S}} \inf \{\sigma: x+\sigma F(\rho, \alpha) \in \mathcal{C}\}
$$

The following condition is necessary and sufficient for $W(x)$ to be finite for all $x \in \mathbb{R}^{N}$. Let $\mathcal{C}^{\circ}$ denote the interior of $\mathcal{C}$.

Condition 3.1 For each $\alpha \in \mathcal{A}$ there exists $\rho \in \mathcal{S}$ such that

$$
F(\rho, \alpha) \in \mathcal{C}^{\circ}
$$

It follows directly from the definition of $W_{\alpha}(x)$ that under this condition $W_{\alpha}(x)<\infty$ for all $x \in \mathbb{R}^{N}$. Since $\mathcal{A}$ is compact, an open covering argument can be used to prove that $W(x)<\infty$ for all $x \in \mathbb{R}^{N}$.

In order to motivate the representation (6), we first consider (7). In this case there is just "true" control for a fixed set of arrival and service rates. It turns out that $W_{\alpha}$ equals the minimum time for a control problem that uses the dynamics defined by the Skorokhod Problem and stops when the origin is reached. However, from the formula for $W_{\alpha}$ it is clear that $W_{\alpha}$ equals the solution to the minimum time problem with the much simpler dynamics $\dot{\phi}(t)=F(\rho(t), \alpha)$ and the stopping set $\mathcal{C}$. Away from the boundary $\partial \mathbb{R}_{+}^{N}$ these two different minimum time problems should satisfy the same Hamilton-Jacobi Bellman equation. 


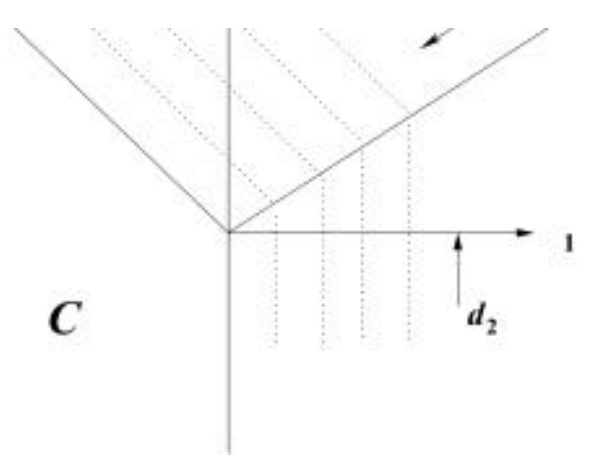

Figure 5: Level sets of $W_{\alpha}$ : Classical boundary conditions

Owing to the constraining dynamics, the first minimum time problem should satisfy a Neumann boundary condition $\left\langle D W_{\alpha}(x), d_{i}\right\rangle=0$ for $i \in I(x)$ on $\partial \mathbb{R}_{+}^{N} \backslash\{0\}$ (in the viscosity sense). It turns out (under Condition 2.1) that the shape of the stopping set $\mathcal{C}$ in the second minimum time problem produces a function whose gradient satisfies this boundary condition, and so by uniqueness one would expect the two minimum time problems to coincide on $\mathbb{R}_{+}^{N}$ [1]. Figure 5 illustrates the situation for a particular two dimensional problem with no control (so that $F(\rho, \alpha)=v$ is a constant). The dotted lines indicate level curves of $W_{\alpha}$, and since these level curves are parallel to $d_{i}$ for $x$ near $\left\{x \in\left(\mathbb{R}_{+}^{2}\right) \backslash\{0\}: x_{i}=0\right\}$, the boundary conditions hold, even in a classical sense.

The situation is not always so simple, as indicated by Figure 6 .

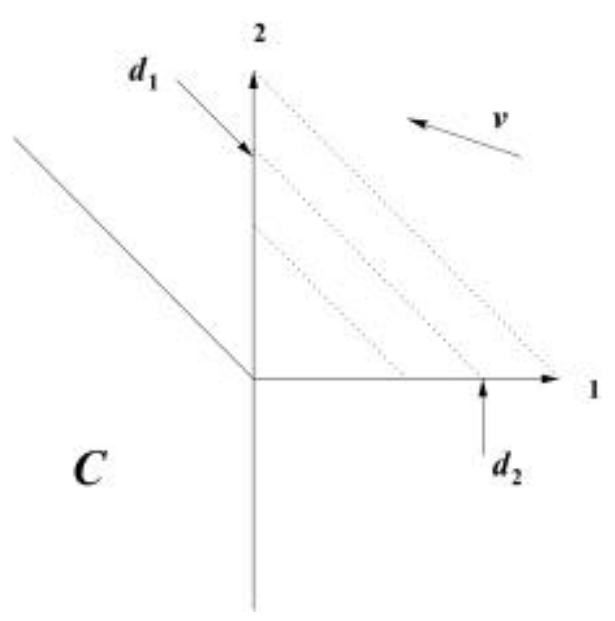

Figure 6: Level sets of $W_{\alpha}$ : Without classical boundary conditions

Here only one boundary condition holds in the classical sense, and this is due to the fact that at the other boundary $v$ points into the interior and away from this boundary. One of the important properties of viscosity solutions is that they allow such relaxations. 
The remarkable fact is that an analogous representation continues to hold even in the game problem, with simply an additional supremization on $\alpha \in \mathcal{A}$. It should be noted that even though the game has value, one cannot permute the $\inf _{\rho \in \mathcal{S}}$ and $\sup _{\alpha \in \mathcal{A}}$ in (6).

In the rest of this section we will prove qualitative properties of $W$ that are needed for the proof that $W$ is the value of the game.

Theorem 3.1 Assume that Conditions 3.1 and 2.1 are satisfied and define $W_{\alpha}(x)$ for $x \in \mathbb{R}^{N}$ and $\alpha \in \mathcal{A}$ by (7). The following conclusions hold.

1. $W_{\alpha}$ is finite and radially linear on $\mathbb{R}^{N}$.

2. For each $x \in \mathbb{R}^{N}$ the infimum in (7) is achieved at some probability vector $\rho$.

3. $W_{\alpha}$ is convex on $\mathbb{R}^{N}$.

4. $W_{\alpha}(x)>0$ for $x \in \mathbb{R}_{+}^{N} \backslash\{0\}$.

Proof: Under Condition 3.1 it is obvious that the cone $\mathcal{C}$ can be reached from any starting point $x$, and so $W_{\alpha}(x)<\infty$, while radially linearity is an immediate consequence of the definition of $W_{\alpha}(x)$. It follows from the compactness of $\mathcal{S}$ that the infimum is achieved in the definition of $W_{\alpha}(x)$. Thus the proofs of parts 1 and 2 are complete.

To prove property 3 we first consider points $x_{1}$ and $x_{2}$ such that $W_{\alpha}\left(x_{1}\right)=$ $W_{\alpha}\left(x_{2}\right) \neq 0$. Let $c$ denote the common value, and let $\rho^{1}$ and $\rho^{2}$ denote minimizing probability vectors in the expression that defines $W_{\alpha}\left(x_{1}\right)$ and $W_{\alpha}\left(x_{2}\right)$, respectively. Thus $x_{i}+c F\left(\rho^{i}, \alpha\right) \in \partial \mathcal{C}$ for $i=1,2$. For $s \in[0,1]$, the convexity of $\mathcal{C}$ implies

$$
\begin{aligned}
& s x_{1}+(1-s) x_{2}+s c F\left(\rho^{1}, \alpha\right)+(1-s) c F\left(\rho^{2}, \alpha\right) \\
& \quad=\left(s x_{1}+(1-s) x_{2}\right)+c F\left(s \rho^{1}+(1-s) \rho^{2}, \alpha\right) \in \mathcal{C} .
\end{aligned}
$$

Since $s \rho^{1}+(1-s) \rho^{2} \in \mathcal{S}$, it follows that

$$
W_{\alpha}\left(s x_{1}+(1-s) x_{2}\right) \leq c=s W_{\alpha}\left(x_{1}\right)+(1-s) W_{\alpha}\left(x_{2}\right) .
$$

We next consider the case of any points $x_{1}$ and $x_{2}$ such that $W_{\alpha}\left(x_{1}\right) \neq 0$ and $W_{\alpha}\left(x_{2}\right) \neq 0$. Let

$$
c=s W_{\alpha}\left(x_{1}\right)+(1-s) W_{\alpha}\left(x_{2}\right) .
$$


Since $W_{\alpha}$ is radially homogeneous,

$$
\begin{aligned}
& W_{\alpha}\left(s x_{1}+(1-s) x_{2}\right) \\
& =W_{\alpha}\left(s \frac{W_{\alpha}\left(x_{1}\right)}{W_{\alpha}\left(x_{1}\right)} x_{1}+(1-s) \frac{W_{\alpha}\left(x_{2}\right)}{W_{\alpha}\left(x_{2}\right)} x_{2}\right) \\
& =W_{\alpha}\left(\left[\frac{s W_{\alpha}\left(x_{1}\right)}{c} \frac{x_{1}}{W_{\alpha}\left(x_{1}\right)}+\frac{(1-s) W_{\alpha}\left(x_{2}\right)}{c} \frac{x_{2}}{W_{\alpha}\left(x_{2}\right)}\right] c\right) \\
& \leq \frac{s W_{\alpha}\left(x_{1}\right)}{c} W_{\alpha}\left(\frac{x_{1}}{W_{\alpha}\left(x_{1}\right)} c\right)+\frac{(1-s) W_{\alpha}\left(x_{2}\right)}{c} W_{\alpha}\left(\frac{x_{2}}{W_{\alpha}\left(x_{2}\right)} c\right) \\
& =s W_{\alpha}\left(x_{1}\right)+(1-s) W_{\alpha}\left(x_{2}\right) .
\end{aligned}
$$

The case where $W_{\alpha}\left(x_{1}\right)$ or $W_{\alpha}\left(x_{2}\right)$ equals zero is similar and omitted.

Finally we must prove property 4 . The Harrison-Reiman condition implies what is called the completely-S condition $([17,4])$, which requires the existence of a vector $v \in \mathbb{R}^{N}$ satisfying $v_{i}>0, i=1, \ldots, N$ and $\langle v, \gamma\rangle>0$ for all $\gamma \in d(0)$. Hence if $y \in \mathcal{C} \backslash\{0\}$ then $\langle v, y\rangle<0$, and so $y \notin \mathbb{R}_{+}^{N}$. This shows that

$$
\mathcal{C} \cap \mathbb{R}_{+}^{N}=\{0\}
$$

and therefore $W_{\alpha}(x)>0$ for all $x \in \mathbb{R}_{+}^{N} \backslash\{0\}$.

Theorem 3.2 Assume that Conditions 3.1 and 2.1 are satisfied and define $W(x)$ for $x \in \mathbb{R}^{N}$ by (6). The following conclusions hold.

1. $W$ is finite and radially linear on $\mathbb{R}^{N}$.

2. $W$ is convex on $\mathbb{R}^{N}$.

3. $W(x)>0$ for $x \in \mathbb{R}_{+}^{N} \backslash\{0\}$.

Proof: It follows from Condition 3.1 that for each $x \in \mathbb{R}^{N} W_{\alpha}(x)$ is bounded uniformly in $\alpha \in \mathcal{A}$. All the claims then follow from the preceding theorem and $W(x)=\sup _{\alpha \in \mathcal{A}} W_{\alpha}(x)$.

Remark. Since $W$ is convex, directional derivatives exist at all points and for all directions.

Theorem 3.3 Assume that Conditions 2.1 and 3.1 are satisfied and define $W(x), V^{-}(x)$ and $V^{+}(x)$ by (6), (4), and (5), respectively. Then for all $x \in \mathbb{R}_{+}^{N}$

$$
W(x)=V^{-}(x)=V^{+}(x) .
$$




\section{Proof of the Representation}

In this section we give the proof of Theorem 3.3. The proof that the differential game has value (i.e., that $\left.V^{-}(x)=V^{+}(x)\right)$ is deferred to the appendix. We first prove some preparatory lemmas. Let

$$
\rho(x, \alpha) \doteq\left\{\rho \in \mathcal{S}: x+W_{\alpha}(x) F(\rho, \alpha) \in \mathcal{C}\right\} .
$$

Lemma 4.1 Assume Condition 3.1. For each $x \in \mathbb{R}_{+}^{N}$ and $\alpha \in \mathcal{A}$ the set $\rho(x, \alpha)$ is nonempty and convex, and moreover the mapping from $\mathbb{R}_{+}^{N} \times \mathcal{A}$ to $\mathcal{S}$ defined by $(x, \alpha) \rightarrow \rho(x, \alpha)$ is upper semicontinuous.

Proof: Fix $x \in \mathbb{R}_{+}^{N}$ and $\alpha \in \mathcal{A}$, and let $\rho^{m}$ come within $1 / m$ of the infimum of

$$
\inf \{\sigma: x+\sigma F(\rho, \alpha) \in \mathcal{C}\}
$$

over $\rho \in \mathcal{S}$. Let $\sigma^{m}$ come within $1 / m$ of the infimum over $\sigma$ when $\rho=\rho^{m}$. By extracting a subsequence, we can assume that $\left(\rho^{m}, \sigma^{m}\right) \rightarrow\left(\rho^{*}, W_{\alpha}(x)\right)$ with $\rho^{*} \in \mathcal{S}$. We claim that $\rho^{*} \in \rho(x, \alpha)$. Indeed, we have

$$
x+W_{\alpha}(x) F\left(\rho^{*}, \alpha\right)=\lim _{m \rightarrow \infty}\left(x+\sigma^{m} F\left(\rho^{m}, \alpha\right)\right) \in \mathcal{C},
$$

which proves that $\rho^{*} \in \rho(x, \alpha)$, and shows that $\rho(x, \alpha)$ is nonempty. Since $\rho \rightarrow F(\rho, \alpha)$ is linear, it follows that $\rho(x, \alpha)$ is also convex.

To prove the upper semicontinuity we first show that $W_{\alpha}(x)$ is jointly continuous in $(x, \alpha)$. Let $\left(x^{i}, \alpha^{i}\right) \rightarrow(x, \alpha)$ as $i \rightarrow \infty$. Under Condition 3.1 , for all $\varepsilon>0$ we can find $\rho \in \mathcal{S}$ such that $x+\left[W_{\alpha}(x)+\varepsilon\right] F(\rho, \alpha) \in$ $\mathcal{C}^{\circ}$. This implies $\lim \sup _{i \rightarrow \infty} W_{\alpha^{i}}\left(x^{i}\right) \leq W_{\alpha}(x)+\varepsilon$, and since $\varepsilon>0$ is arbitrary $\lim \sup _{i \rightarrow \infty} W_{\alpha^{i}}\left(x^{i}\right) \leq W_{\alpha}(x)$. Let $\rho^{i} \in \rho\left(x^{i}, \alpha^{i}\right)$. By extracting a subsequence, we can assume that $W_{\alpha^{i}}\left(x^{i}\right) \rightarrow M$ and $\rho^{i} \rightarrow \rho \in \mathcal{S}$. Taking the limit as $i \rightarrow \infty$ in

$$
x^{i}+W_{\alpha^{i}}\left(x^{i}\right) F\left(\rho^{i}, \alpha^{i}\right) \in \mathcal{C}
$$

gives

$$
x+M F(\rho, \alpha) \in \mathcal{C},
$$

and therefore $\liminf \inf _{i \rightarrow \infty} W_{\alpha^{i}}\left(x^{i}\right) \geq W_{\alpha}(x)$. We conclude that $W_{\alpha}(x)$ is jointly continuous in $(x, \alpha)$.

Next let $\left(x^{i}, \alpha^{i}\right) \rightarrow(x, \alpha)$ as $i \rightarrow \infty$, and let $\rho^{i} \in \rho\left(x^{i}, \alpha^{i}\right)$. We must show that $\rho^{i} \rightarrow \rho^{*}$ implies $\rho^{*} \in \rho(x, \alpha)$. Using the continuity of $W_{\alpha}(x)$,

$$
x+W_{\alpha}(x) F\left(\rho^{*}, \alpha\right)=\lim _{i \rightarrow \infty}\left(x^{i}+W_{\alpha^{i}}\left(x^{i}\right) F\left(\rho^{i}, \alpha^{i}\right)\right) \in \mathcal{C} .
$$


We conclude that $\rho^{*} \in \rho(x, \alpha)$, and therefore $(x, \alpha) \rightarrow \rho(x, \alpha)$ is upper semicontinuous.

Lemma 4.2 Assume that Conditions 2.1 and 3.1 are satisfied and define $W(x)$ for $x \in \mathbb{R}^{N}$ by (6). Consider any point $x \in \mathbb{R}_{+}^{N} \backslash\{0\}$ and let $v \in \mathbb{R}^{N}$ be such that $x+W(x) v \in \mathcal{C}$. Then

$$
D_{v} W(x) \leq-1
$$

Proof: Since $x+W(x) v \in \mathcal{C}$ it follows that $W(x+W(x) v)=0$. The convexity of $W$ then implies that for any $a \in(0, W(x))$

$$
\begin{aligned}
W(x+a v) & \leq \frac{a}{W(x)} W(x+W(x) v)+\left(1-\frac{a}{W(x)}\right) W(x) \\
& \leq\left(1-\frac{a}{W(x)}\right) W(x) .
\end{aligned}
$$

It follows that

$$
D_{v} W(x) \doteq \lim _{a \downarrow 0} \frac{W(x+a v)-W(x)}{a} \leq-1
$$

Define

$$
B(x) \doteq\{F(\rho(x, \alpha), \alpha): \alpha \in \mathcal{A}\} .
$$

These are the velocities that are optimal (for the true controller) at $x$ for the control problem $W_{\alpha}(x)$ for some $\alpha \in \mathcal{A}$.

Lemma 4.3 Assume that Conditions 2.1 and 3.1 are satisfied and define $W(x)$ for $x \in \mathbb{R}^{N}$ by (6). Then for any $x \in \mathbb{R}_{+}^{N}$ and $v \in B(x)$ we have $x+W(x) v \in \mathcal{C}$.

Proof: Suppose that $v \in F(\rho(x, \alpha), \alpha)$ for some $\alpha \in \mathcal{A}$. We know that

$$
x+W_{\alpha}(x) v \in \mathcal{C} \text { and } W_{\alpha}(x) \leq W(x) .
$$

If $v \in \mathcal{C}$ then we are done, since $\mathcal{C}$ is a cone with vertex at the origin. Now $x \in \mathbb{R}_{+}^{N}$ implies that $v=v_{1}+v_{2}$, where $v_{1} \in-\mathbb{R}_{+}^{N}$ and $v_{2} \in \mathcal{C}$. Thus we need only show $-\mathbb{R}_{+}^{N} \subset \mathcal{C}$. Since $\mathcal{C}$ is a convex cone, to show this it is enough to prove that $-e_{i} \in \mathcal{C}$ for each $i=1, \ldots, N$. 
Let the vectors $\left\{d_{i}^{*}, i=1, \ldots, N\right\}$ be defined by

$$
\left\langle d_{i}, d_{j}^{*}\right\rangle=\left\{\begin{array}{ll}
1 & i=j \\
0 & i \neq j
\end{array} .\right.
$$

Condition 2.1 implies the vectors $\left\{d_{i}, i=1, \ldots, N\right\}$ are linearly independent, and so this is well defined. The vectors $\left\{d_{i}^{*}, i=1, \ldots, N\right\}$ provide an external representation for $\mathcal{C}$ in that

$$
\mathcal{C}=\left\{y \in \mathbb{R}^{N}:\left\langle y, d_{i}^{*}\right\rangle \leq 0, i=1, \ldots, N\right\} .
$$

Thus $-e_{i} \in \mathcal{C}$ will follow if we show $\left\langle e_{i}, d_{j}^{*}\right\rangle \geq 0$ for $j=1, \ldots, N$. Let $D$ be the matrix whose $i$ th column is $d_{i}$. Then $D^{-1}$ is the matrix whose $j$ th row is $d_{j}^{*}$. We can write $D=I-A$, where $A$ is nonnegative. Under Condition 2.1 the spectral radius of $A$ is less than one, and so we can express $D^{-1}$ as $\sum_{\ell=0}^{\infty} A^{\ell}$. This shows that $D^{-1}$ is nonnegative, and completes the proof of the lemma.

We recall the definition of the projected velocity given in (1) and $I(x) \doteq$ $\left\{i: x_{i}=0\right\}$ for $x \in \mathbb{R}_{+}^{N}$.

Lemma 4.4 Assume that Conditions 2.1 and 3.1 are satisfied and define $W(x)$ for $x \in \mathbb{R}^{N}$ by (6). Let $x \in \mathbb{R}_{+}^{N}$ be given. Let $y \leq x$ componentwise, and assume $y \notin \mathcal{C}$ (so that $W(y)>0$ ). Let $v \in B(y)$, and suppose there exist $a_{i} \geq 0, i \in I(x)$ such that

$$
\left\langle v+\sum_{i \in I(x)} a_{i} d_{i}, e_{j}\right\rangle=0, \quad j \in I(x) .
$$

Let $q=v+\sum_{i \in I(x)} a_{i} d_{i}$. Then

$$
D_{q} W(y) \leq-1
$$

Proof: By Lemma 4.2 it is enough to show that $y+W(y) q \in \mathcal{C}$. According to the last lemma $y+W(y) v \in \mathcal{C}$, and so we can express $(y / W(y))+v$ as $-\sum_{i=1}^{N} \bar{a}_{i} d_{i}$ for some constants $\bar{a}_{i} \geq 0, i=1, \ldots, N$. To prove

$$
\begin{aligned}
y+W(y) q & =W(y)\left[(y / W(y))+v+\sum_{i \in I(x)} a_{i} d_{i}\right] \\
& =W(y)\left[-\sum_{i=1}^{N} \bar{a}_{i} d_{i}+\sum_{i \in I(x)} a_{i} d_{i}\right] \\
& \in \mathcal{C},
\end{aligned}
$$


it is therefore enough to show that $\bar{a}_{i} \geq a_{i}$ for $i \in I(x)$.

Since $v=-(y / W(y))-\sum_{i=1}^{N} \bar{a}_{i} d_{i}$, the equations (8) can be rewritten as

$$
\left\langle-(y / W(y))-\sum_{i=1}^{N} \bar{a}_{i} d_{i}+\sum_{i \in I(x)} a_{i} d_{i}, e_{j}\right\rangle=0
$$

for $j \in I(x)$. Let $M$ denote the cardinality of $I(x)$. We recall that $\left\langle d_{j}, e_{i}\right\rangle \leq 0$ if $i \neq j$ and $y_{i} \leq x_{i} \leq 0$ for $i \in I(x)$. As a consequence, we can rewrite this system of $M$ equations as

$$
\left(I-D_{M}\right) r=q,
$$

where $I$ is the $M \times M$ identity matrix, $D_{M}$ is non-negative with spectral radius less than $1, r_{j}=a_{j}-\bar{a}_{j}$ for $j \in I(x)$, and $q_{i}=\sum_{j \notin I(x)} \bar{a}_{j}\left\langle d_{j}, e_{i}\right\rangle+$ $\left(y_{i} / W(y)\right) \leq 0$ for each $i \in I(x)$. Since each component of $r=\left(\sum_{\ell=0}^{\infty} D_{M}^{\ell}\right) q$ is obviously nonpositive, we conclude that $a_{i} \leq \bar{a}_{i}$ for $i \in I(x)$.

In the proof of Theorem 3.3 we will need to construct a nearly optimal strategy for the minimizing player to prove that $V^{-}(x) \leq W(x)$. If $W$ were smooth then such a strategy would be easy to construct. However, since $W$ is only convex it must be mollified to construct this policy, and this mollification in turn complicates the construction of the optimal control on the boundary. In the lemma that follows we apply the previous lemma to deal with this issue.

Lemma 4.5 Assume that Conditions 2.1 and 3.1 are satisfied and define $W(x)$ for $x \in \mathbb{R}^{N}$ by (6). Let $\gamma>0$ be given. Then there exists a convex, continuously differentiable and radially linear function $W_{\gamma}: \mathbb{R}^{N} \rightarrow[0, \infty)$ such that for all $x \in \mathbb{R}_{+}^{N}, \alpha \in \mathcal{A}$, and $\rho \in \rho(x, \alpha)$,

$$
\left|W_{\gamma}(x)-W(x)\right| \leq \gamma W(x)
$$

and

$$
\left\langle\pi(x, F(\rho, \alpha)), D W_{\gamma}(x)\right\rangle \leq-(1-\gamma) .
$$

Proof: Fix $\gamma>0$. We begin by noting a relation between directional derivatives and subdifferentials for convex functions. Fix $x \in \mathbb{R}_{+}^{N}$, and let $\partial W(x)$ denote the set of subdifferentials of $W$ at $x$. Then for any $v \in \mathbb{R}^{N}$ and any $q \in \partial W(x),\langle q, v\rangle \leq D_{v} W(x)$. According to Lemmas 4.1, 4.2, and 4.3 , for each $\alpha \in \mathcal{A}$ and $\rho \in \rho(x, \alpha)$ we have $D_{F(\rho, \alpha)} W(x) \leq-1$, and therefore for all such $\alpha$ and $\rho$

$$
\langle q, F(\rho, \alpha)\rangle \leq-1
$$


for all $q \in \partial W(x)$.

We next mollify the function $W$. Define the convex set $G \doteq\{x: W(x) \leq$ $1\}$. For $a>0$ define the translation $G_{a} \doteq\{y=x+a(1, \ldots, 1): x \in G\}$, and for $\delta>0$ consider the $\delta$-fattening $G_{a}^{\delta} \doteq\left\{y:\|y-x\| \leq \delta\right.$ for some $\left.x \in G_{a}\right\}$. Since $0 \in G^{\circ}$, we can assume without loss that $a$ is small enough that the origin is contained in the interior of $G_{a}^{\delta}$. As we will see, the translation is needed to ensure that the fattening does not interfere with the boundary conditions that are required of the mollification. Finally, let

$$
W_{a}^{\delta}(x) \doteq \inf \left\{c \geq 0: x \in \partial\left(c G_{a}^{\delta}\right)\right\} .
$$

The construction is illustrated in Figure 7.

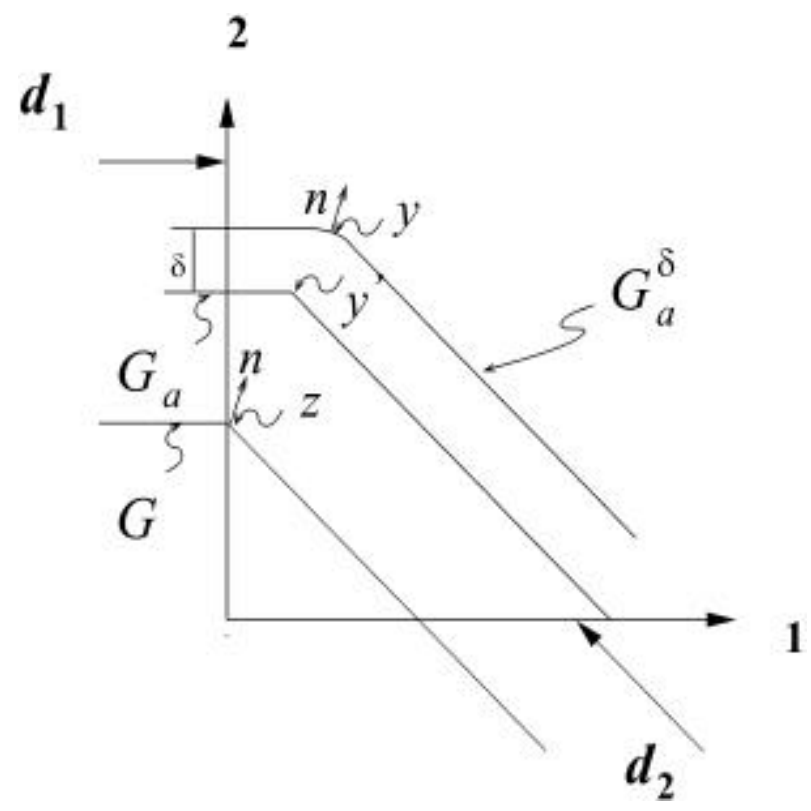

Figure 7: Construction of $G_{a}^{\delta}$

It is easy to check that $W_{a}^{\delta}$ is finite and convex. Also, it is well known that $G_{a}^{\delta}$ has a $C^{1}$ boundary for each $\delta>0$, and thus $W_{a}^{\delta}$ is continuously differentiable on $\mathbb{R}_{+}^{N} \backslash\{0\}$. We first compute the gradient of $W_{a}^{\delta}$. Fix any point $x \in \mathbb{R}_{+}^{N} \backslash\{0\}$ and let $n$ be the outward normal to $G_{a}^{\delta}$ at $y \doteq x / W_{a}^{\delta}(x)$. 
Since $W_{a}^{\delta}$ is radially linear the gradient of $W_{a}^{\delta}(x)$ must be proportional to $n$, which means there must be a supporting hyperplane of the form $\langle x, r n\rangle$ to $W_{a}^{\delta}$ at $x$ (here we use the fact that $W_{a}^{\delta}(0)=0$ ). Thus using the equality $W_{a}^{\delta}(x)=\langle x, r n\rangle$, we find that

$$
D W_{a}^{\delta}(x)=r n=\left(\frac{W_{a}^{\delta}(x)}{\langle x, n\rangle}\right) n=\left(\frac{1}{\langle y, n\rangle}\right) n .
$$

Let $y^{\prime}$ be the unique point in $G_{a}$ that is exactly distance $\delta$ from $y$, and let $z=y-a(1, \ldots, 1)$. Then $n$ is also an outward normal to $G$ at $z$, and an analogous calculation to the one just given shows that for any point of the form $b z, b \in(0, \infty),(1 /\langle z, n\rangle) n$ is a subdifferential to $W$ at $b z$. Therefore

$$
D W_{a}^{\delta}(x)=\left(\frac{\langle z, n\rangle}{\langle y, n\rangle}\right) q
$$

where $q$ is a subdifferential to $W$ at $z$. We can make $|y-z|$ as small as desired by choosing $a>0$ and $\delta>0$ small. Let $\rho \in \rho(x, \alpha)$. Since $\langle y, n\rangle$ is uniformly bounded from below away from zero, for all sufficiently small $a>0$ and $\delta>0$ equation (11) implies

$$
\left\langle F(\rho, \alpha), D W_{a}^{\delta}(x)\right\rangle \leq-(1-\gamma) .
$$

Observe that conditions (8) characterize $\pi(x, F(\rho, \alpha))$. Thus if we knew that $z \leq y$ (componentwise) then

$$
\left\langle\pi(x, F(\rho, \alpha)), D W_{a}^{\delta}(x)\right\rangle \leq-(1-\gamma)
$$

would also follow from Lemma 4.4. However, $z \leq y$ follows easily by fixing $a>0$ and then choosing $\delta \in(0, a)$. Finally, it is also easy to check that $G$ and $G_{a}^{\delta}$ can be made arbitrarily close in the Hausdorff topology, which immediately implies

$$
\left|W_{a}^{\delta}(x)-W(x)\right| \leq \gamma W(x)
$$

when $a$ and $\delta$ are small. The lemma now follows by taking $W_{\gamma}=W_{a}^{\delta}$ for suitable $a>0$ and $\delta>0$.

In the proof of Theorem 3.3 we will use a verification argument to show $V^{-}(x) \leq W_{\gamma}(x)$ plus a small error. The use of feedback controls for the minimizing player would be problematic. The next lemma will allow the use of piecewise constant controls and thereby simplify the proof. The lemma is an immediate consequence of the continuity of $D W_{\gamma}(x)$ for $\gamma>0$ and $x \neq 0$. 
Lemma 4.6 Assume that Conditions 2.1 and 3.1 are satisfied and for $\gamma>0$ define $W_{\gamma}(x)$ for $x \in \mathbb{R}^{N}$ by Lemma 4.5. Then there is $\nu>0$ such that for all $z \in \mathbb{R}_{+}^{N}$ with $\|z\|=1$, all $y$ with $\|z-y\| \leq \nu$, all $\alpha \in \mathcal{A}$, and all $\rho \in \rho(z, \alpha)$,

$$
\left\langle\pi(z, F(\rho, \alpha)), D W_{\gamma}(y)\right\rangle \leq-(1-2 \gamma) .
$$

Proof of Theorem 3.3: We first prove that $W(x) \leq V^{+}(x)$. Fix $x \in$ $\mathbb{R}_{+}^{d} \backslash\{0\}$ and $\alpha \in \mathcal{A}$. Let $\rho \in N$ be any open loop control, and let $\tau_{x}>0$ be the corresponding first time that the origin is reached by the solution to

$$
\dot{\phi}(t)=\pi(\phi(t), F(\rho(t), \alpha)), \phi(0)=x .
$$

If $\tau_{x}=\infty$ there is nothing to prove, and so we assume $\tau_{x}<\infty$. Using the definition of the Skorokhod Problem, there exist $a_{i}(t) \geq 0, i=1, \ldots, N, t \in$ $\left[0, \tau_{x}\right]$ such that

$$
\dot{\phi}(t)=F(\rho(t), \alpha)+\sum_{i=1}^{N} a_{i}(t) d_{i}
$$

for almost every $t \in\left[0, \tau_{x}\right]$. Integrating over $\left[0, \tau_{x}\right]$ and using the definition $\bar{\rho} \doteq \frac{1}{\tau_{x}} \int_{0}^{\tau_{x}} \rho(t) d t$, we find that

$$
-x=\tau_{x} F(\bar{\rho}, \alpha)-\omega
$$

for some $\omega \in \mathcal{C}$, and so $x+\tau_{x} F(\bar{\rho}, \alpha) \in \mathcal{C}$. The definition of $W_{\alpha}(x)$ then implies $\tau_{x} \geq W_{\alpha}(x)$. Since $\theta[\rho](t)=\alpha$ is a legitimate strategy to use in the definition of $V^{+}(x)$ and $\rho \in N$ is arbitrary, it follows that $V^{+}(x) \geq W_{\alpha}(x)$ for all $\alpha \in \mathcal{A}$. Taking the supremum on $\alpha \in \mathcal{A}$ gives $V^{+}(x) \geq W(x)$.

We next prove $W(x) \geq V^{-}(x)$. Let $\gamma \in(0,1 / 2)$, and let $\nu>0$ be given according to Lemma 4.6. Fix $x \in \mathbb{R}_{+}^{d} \backslash\{0\}$ and let the open loop control $\alpha \in M$ be given. We recursively construct a strategy $\delta \in \Delta$ as follows. Given a point of the form $x_{i} \neq 0$ (with $x_{0}=x$ ) and corresponding times $\tau_{i}$ (with $\left.\tau_{0}=0\right)$, we consider the normalized version $z_{i}=x_{i} /\left\|x_{i}\right\|$. Let $\rho^{*}(x, \alpha)$ be any single-valued and measurable selection from $\rho(x, \alpha)$. We define $\delta[\alpha](t)$ for $t \in\left[\tau_{i}, \tau_{i+1}\right)$ to be $\rho^{*}\left(z_{i}, \alpha(t)\right)$, where $\tau_{i+1}>\tau_{i}$ is defined by

$$
\inf \left\{t \geq \tau_{i}:\|\phi(t) /\| \phi(t)\left\|-z_{i}\right\| \geq \nu\right\} \wedge \inf \left\{t \geq \tau_{i}: \phi(t)=0\right\}
$$

where

$$
\dot{\phi}(t)=\pi\left(\phi(t), F\left(\rho^{*}\left(z_{i}, \alpha(t)\right), \alpha(t)\right)\right), \quad \phi\left(\tau_{i}\right)=x_{i} .
$$

Since the speed $\|\dot{\phi}(t)\|$ is uniformly bounded from above, it is easy to check that $\inf \left\{t \geq \tau_{i}:\|\phi(t) /\| \phi(t)\left\|-z_{i}\right\| \geq \nu\right\}-\tau_{i}$ is uniformly bounded away 
from zero if $x_{i}$ is in a closed set that does not contain the origin. We will make use of the fact that for any $x \neq 0$ and any $v \pi(x, v)=\pi(x /\|x\|, v)$. According to Lemma 4.6,

$$
\left\langle D W_{\gamma}(\phi(t)), \dot{\phi}(t)\right\rangle=\left\langle D W_{\gamma}(\phi(t)), \pi\left(\phi(t), F\left(\rho^{*}\left(z_{i}, \alpha(t)\right), \alpha(t)\right)\right)\right\rangle \leq-1+2 \gamma
$$

for almost every $t$ prior to the first time $\phi$ hits the origin, and therefore for all such times

$$
W_{\gamma}(\phi(t))-W_{\gamma}(x)=\int_{0}^{t}\left\langle D W_{\gamma}(\phi(t)), \dot{\phi}(t)\right\rangle d s \leq-t(1-2 \gamma) .
$$

We conclude that

$$
W_{\gamma}(\phi(t)) \leq W_{\gamma}(x)-t(1-2 \gamma)
$$

and therefore $\phi$ reaches the origin by time $W_{\gamma}(x) /(1-2 \gamma)$. This implies $V^{-}(x) \leq W_{\gamma}(x) /(1-2 \gamma)$, and since $\gamma>0$ is arbitrary, that $V^{-}(x) \leq W(x)$.

Thus we have shown that $V^{-}(x) \leq W(x) \leq V^{+}(x)$. The proof that $V^{-}(x)=V^{+}(x)$ is based on a uniqueness result for the corresponding PDE, and is presented in the Appendix. This completes the proof of the theorem.

\section{Synthesis of Controls}

The "true" controls used to prove $W(x) \geq V^{-}(x)$ in the proof of Theorem 3.3 are not very useful, since they require knowledge of the control that nature applies at all times. In this section we will formally discuss how to construct controls that are optimal (or nearly optimal), and which depend only on the state of the network. A rigorous proof will appear elsewhere.

Formally, $W=V^{-}=V^{+}$is the solution to the equation

$$
\sup _{\alpha \in \mathcal{A}} \inf _{\rho \in \mathcal{S}}[\langle D W(x), F(\rho, \alpha)\rangle+1]=0, \quad x \in\left(\mathbb{R}_{+}^{N}\right)^{\circ},
$$

together with the boundary conditions

$$
\left\langle D W(x), d_{i}\right\rangle=0, \quad i \in I(x), x \in \partial \mathbb{R}_{+}^{N} \backslash\{0\}, \quad W(0)=0 .
$$

Since $F$ is affine in each variable seperately and $\mathcal{A}$ and $\mathcal{S}$ are compact and convex, [19, Corollary 37.6.2] implies that the sup and inf in (13) can be interchanged (i.e., one expects the game to have value).

Since $W$ is not necessarily smooth we cannot expect a classical sense solution to (13)-(14), and so one must consider a weak sense solution, e.g., 
viscosity solutions. Because $W$ is convex, the set of subdifferentials to $W$ at $x$ (denoted $D^{-} W(x)$ ) is never empty. It follows from the characterization of viscosity solutions (see the Appendix) that for any $q \in D^{-} W(x)$ there exists at least one saddle point $(\rho(q), \alpha(q))$ such that

$$
\sup _{\alpha \in \mathcal{A}}\langle q, F(\rho(q), \alpha)\rangle \leq-1 .
$$

Let $R(q)$ denote the set of all points $\rho \in \mathcal{S}$ which have this property. It is easy to check that this set-valued function is upper semicontinuous: $q_{n} \rightarrow q$, $\rho_{n} \rightarrow \rho$ and $\rho_{n} \in R\left(q_{n}\right)$ implies $\rho \in R(q)$. At each point $x \in \mathbb{R}_{+}^{N}$ we define a set of controls $S(x) \subset \mathcal{S}$ by

$$
S(x)=\cup_{q \in D^{-} W(x)} R(q) .
$$

Note that since $x \rightarrow D^{-} W(x)$ and $q \rightarrow R(q)$ are upper semicontinuous, so is the composition $S(x)$, and that the radial linearity of $W$ implies a radial homogeneity of $S: S(a x)=S(x)$ for all $x \in \mathbb{R}_{+}^{N}$ and $a \in(0, \infty)$. The set of conjectured controls for $x$ in the interior is then $S(x)$.

However, when on the boundary we must be more careful. As can easily be seen by considering two dimensional examples, there is an important distinction depending in whether the boundary condition holds in a classical sense or not. The following conjectures for the form of the optimal control are based on the analysis of two dimensional examples, and have not been verified in any generality. Let us first consider the case of a point $x$ where $I(x)=i$ for a single value $i$. In this case the classical sense formulation of the boundary condition is $\left\langle D W(x), d_{i}\right\rangle=0$. If this condition holds, it means that all optimally controlled trajectories push into the boundary, and that any selection from $S(x)$ is optimal. If however $\left\langle D W(x), d_{i}\right\rangle \neq 0$, then even if some elements from $S(x)$ lead to trajectories that push into the boundary, we must restrict ourselves to only those for which the saddle point dynamics do not push strictly into the boundary. If the boundary condition is not valid in the classical sense, then we conjecture that this set is always nonempty. Analogous considerations hold for the points at the intersection of two or more faces. In general, choosing a control for which the saddle point dynamics push into a face is only allowed when the corresponding boundary condition holds in the classical sense.

\section{Appendix}

In this appendix we will prove that the game has value, i.e., that $V^{+}(x)=$ $V^{-}(x)$ for all $x \in \mathbb{R}_{+}^{N}$. A key ingredient is a uniqueness result for the partial 
differential equation (PDE) that $V^{+}$and $V^{-}$should satisfy. An excellent general reference for the theory of viscosity solutions of first order nonlinear PDE is the book [2]. The particular results we will need can be found in [1] (see also [9]).

For $q \in \mathbb{R}^{N}$ define

$$
\begin{aligned}
H(q) & =\max _{\alpha \in \mathcal{A}} \min _{\rho \in \mathcal{S}}[\langle q, F(\rho, \alpha)\rangle+1] \\
& =\min _{\rho \in \mathcal{S}} \max _{\alpha \in \mathcal{A}}[\langle q, F(\rho, \alpha)\rangle+1],
\end{aligned}
$$

where the two expressions on the right hand side are equal since $F(\rho, \alpha)$ is affine in each variable separately and $\mathcal{S}$ and $\mathcal{A}$ are convex and compact. Consider a Lipschitz continuous function $V: \mathbb{R}_{+}^{N} \rightarrow \mathbb{R}$, and for a continuously differentiable function $g: \mathbb{R}^{N} \rightarrow \mathbb{R}$ let $y$ be a local maximum (respectively, minimum) of

$$
x \rightarrow V(x)-g(x)
$$

Then $V$ is called a viscosity subsolution (respectively, viscosity supersolution) to (13) and (14) if

$$
\begin{gathered}
H(D g(y)) \vee \max _{i \in I(y)}\left\langle D g(y), d_{i}\right\rangle \geq 0 \\
\left(H(D g(y)) \wedge \min _{i \in I(y)}\left\langle D g(y), d_{i}\right\rangle \leq 0\right),
\end{gathered}
$$

and

$$
V(0) \leq 0, \quad(V(0) \geq 0) .
$$

We henceforth drop the adjective "viscosity," and note that a function that is both a sub and supersolution is called a solution.

Recall that $V: \mathbb{R}_{+}^{N} \rightarrow \mathbb{R}$ is said to be radially linear if $V(a x)=a V(x)$ for all $x \in \mathbb{R}_{+}^{N}$ and $a \in[0, \infty)$. According to [1, Theorem 4.3], there is only one function $V$ satisfying the following conditions: (i) $V$ is a viscosity solution to (15)-(17), (ii) $V$ is Lipschitz continuous and radially linear, and (iii) $V(x)>0$ for $x \in\left(\mathbb{R}_{+}^{N}\right) \backslash\{0\}$. Suppose that $V^{+}$and $V^{-}$satisfy conditions (ii) and (iii) of the last sentence. Then standard arguments based on dynamic programming can be used to show that (i) holds ([1, Theorem 3.2] and [2, Chapter VIII]). Thus $V^{+}=V^{-}$will follow if we can prove that (ii) and (iii) hold for both $V^{+}$and $V^{-}$.

Assume for now that $V^{+}$is uniformly bounded on bounded sets. It follows from Theorem 3.3 that $0 \leq V^{-}(x) \leq V^{+}(x) \leq \infty$. It is also immediate from the definitions that both $V^{+}(x)$ and $V^{-}(x)$ are radially linear, 
and that $V^{+}(x) \wedge V^{-}(x)>0$ for $x \in\left(\mathbb{R}_{+}^{N}\right) \backslash\{0\}$. Thus if $V^{+}$is uniformly bounded on bounded sets, all that needs to be shown is that $V^{+}$and $V^{-}$ are Lipschitz continuous. We give the proof for $V^{+}$, and note that the proof for $V^{-}$is analogous. Let $M \doteq \max _{y:\|y\|=1} V^{+}(y)$, and assume for now that $M<\infty$. Owing to the radial linearity, $V^{+}(x) \leq M\|x\|$. Fix points $x, y \in \mathbb{R}_{+}^{N}$ and $\varepsilon>0$. Let $K<\infty$ be the Lipschitz constant of the Skorokhod Map defined in Section 2. We claim that $V^{+}$is Lipschitz continuous with constant $M K$. The proof adapts a standard argument [2]. Choose $\bar{\theta} \in \Theta$ such that $V^{+}(x) \leq \inf _{\rho \in N} C_{x}(\rho, \bar{\theta}[\rho])+\varepsilon / 2$. Since $\bar{\theta}$ is suboptimal at $y, V^{+}(y) \geq \inf _{\rho \in N} C_{y}(\rho, \bar{\theta}[\rho])$, and hence there is $\bar{\rho}$ such that $V^{+}(y) \geq C_{y}(\bar{\rho}, \bar{\theta}[\bar{\rho}])-\varepsilon / 2$. Note that also $V^{+}(x) \leq C_{x}(\bar{\rho}, \bar{\theta}[\bar{\rho}])+\varepsilon / 2$, and hence

$$
V^{+}(x)-V^{+}(y) \leq C_{x}(\bar{\rho}, \bar{\theta}[\bar{\rho}])-C_{y}(\bar{\rho}, \bar{\theta}[\bar{\rho}])+\varepsilon .
$$

If $C_{y}(\bar{\rho}, \bar{\theta}[\bar{\rho}]) \geq C_{x}(\bar{\rho}, \bar{\theta}[\bar{\rho}])$ (i.e., it takes longer to reach the origin from $y$ than $x)$ then of course $V^{+}(x)-V^{+}(y) \leq \varepsilon$. On the other hand, if $C_{y}(\bar{\rho}, \bar{\theta}[\bar{\rho}]) \leq$ $C_{x}(\bar{\rho}, \bar{\theta}[\bar{\rho}])$ then we can stop the process that was started at $x$ at time $\sigma \dot{=}$ $C_{y}(\bar{\rho}, \bar{\theta}[\bar{\rho}])$. If we let $\phi^{x}(t)$ and $\phi^{y}(t)$ denote the processes started at the points $x$ and $y$, then the Lipschitz property of the Skorokhod Map implies $\left\|\phi^{x}(\sigma)-\phi^{y}(\sigma)\right\| \leq K\|x-y\|$. Since $\phi^{y}(\sigma)=0$, this means that $\left\|\phi^{x}(\sigma)\right\| \leq$ $K\|x-y\|$. We can now use dynamic programming to argue that $V^{+}(x) \leq$ $\sigma+V^{+}\left(\phi^{x}(\sigma)\right)+\varepsilon / 2 \leq \sigma+M K\|x-y\|+\varepsilon / 2$, and thus $V^{+}(x)-V^{+}(y) \leq$ $M K\|x-y\|+\varepsilon$. Combining the two cases and using that $\varepsilon>0$ is arbitrary, it follows that $V^{+}(x)-V^{+}(y) \leq M K\|x-y\|$ for all $x, y \in \mathbb{R}_{+}^{N}$.

With the proof that $V^{+}$and $V^{-}$are Lipschitz continuous complete, all that remains is to prove that $V^{+}$is uniformly bounded on bounded sets. Under Condition 2.1, it was shown in [8, Lemma 2.1, Theorem 2.1, and page 60] that there is a compact, convex set $B \subset \mathbb{R}^{N}$ with the following properties:

1. $0 \in B^{\circ}$,

2. if $z \in \partial B$ and $n$ is an outward normal to $B$ at $z$, then $\left|\left\langle z, e_{i}\right\rangle\right| \leq 1$ implies $\left\langle n, d_{i}\right\rangle=0$,

3. if $z \in \partial B$ and $n$ is an outward normal to $B$ at $z$, then $\left\langle z, e_{i}\right\rangle\left\langle n, d_{i}\right\rangle \geq 0$.

By considering sets of the form $B^{\delta} \doteq\{y:\|y-x\| \leq \delta$ for some $x \in B\}$ (with $\delta>0$ ), it is easy to verify that without loss we can assume $B$ has a continuously differentiable boundary. Define the function $R: \mathbb{R}^{N} \rightarrow[0, \infty)$ by

$$
R(x) \doteq \inf \{c: x \in \partial(c B)\}
$$


¿From the convexity, properties 1 and 2 listed above, and the smoothness of $\partial B$, it follows that $R$ is continuously differentiable save at $x=0$, and that for $x \in \mathbb{R}_{+}^{N} \backslash\{0\}$

$$
\left\langle D R(x), d_{i}\right\rangle=0 \text { if } i \in I(x) .
$$

Now fix any point $x \in \mathbb{R}_{+}^{N} \backslash\{0\}$, and let $z \in \partial B$ satisfy $z=a x$ for some $a \in(0, \infty)$. If $n$ is the corresponding outward normal to $B$ at $z$, then $D R(x)=b n$ for some $b \in(0, \infty)$. According to properties 2 and 3 above, $\left\langle D R(x), d_{i}\right\rangle \geq 0$. Let the vectors $d_{i}^{*}, i=1, \ldots, N$ be defined by $\left\langle d_{i}, d_{j}^{*}\right\rangle=\delta_{i j}$, where $\delta_{i j}$ is 1 if $i=j$ and 0 otherwise. These vectors are well defined, since Condition 2.1 implies the linear independence of $\left\{d_{i}, i=1, \ldots, N\right\}$. Writing $\operatorname{DR}(x)=\sum_{i=1}^{N} c_{i} d_{i}^{*}$, it follows from $\left\langle D R(x), d_{i}\right\rangle \geq 0$ that $c_{i} \geq 0$ for $i=1, \ldots, N$. We now apply Condition 3.1. It follows from this condition that for each $\alpha \in \mathcal{A}$ there is $\rho \in \mathcal{S}$ and $c>0$ such that

$$
\langle D R(x), F(\rho, \alpha)\rangle \leq-c \text {. }
$$

Since $\mathcal{A}$ is compact, an open covering argument shows the existence of $c>0$ such that

$$
\max _{\alpha \in \mathcal{A}} \min _{\rho \in \mathcal{S}}\langle D R(x), F(\rho, \alpha)\rangle \leq-c .
$$

Finally, the radial linearity of $R$, the continuity of $D R(x)$, and another open covering argument that uses the compactness of $\partial B \cap \mathbb{R}_{+}^{N}$ shows that $c>0$ can be selected so that (19) holds for all $x \in \mathbb{R}_{+}^{N} \backslash\{0\}$.

Equations (18) and (19) imply that $R / c$ is a (classical) supersolution to (13) and (14). Standard arguments based on dynamic programming can then be used to show that $V^{+}(x) \leq R(x) / c$. (See, for example, the proof

of Theorem 3.3.) This completes the proof that $V^{+}(x)=V^{-}(x)$ for all $x \in \mathbb{R}_{+}^{N}$.

\section{References}

[1] R. Atar and P. Dupuis. A differential game with constrained dynamics and viscosity solutions of a related HJB equation. to appear.

[2] M. Bardi and I. Capuzzo-Dolcetta. Optimal Control and Viscosity Solutions of Hamilton-Jacobi-Bellman Equations. Birkhäuser, 1997.

[3] T. Basar and P. Bernhard. H Optimal Control and Related Minimax Design Problems: A Dynamic Game Approach. Birkhauser, 1991. 
[4] A. Bernard and A. El Kharroubi. Régulation de processus dans le premier orthant de $\mathbb{R}^{n}$. Stochastics and Stochastics Reports, 34:149$167,1991$.

[5] D. Bertsimas, I. Paschalidis, and J. Tsitsiklis. Optimization of multiclass queueing networks: polyhedral and nonlinear characterizations of achievable performance. Annals of Applied Probability, 4:43-75, 1994.

[6] A. Budhiraja and P. Dupuis. Simple necessary and sufficient conditions for the stability of constrained processes. SIAM J. on Applied Math., 59:1686-1700, 1999.

[7] M.V. Day, J. Hall, J. Menendez, D. Potter, and I. Rothstein. Robust optimal service analysis of single-server re-entrant queues. Technical report, VPI \& SU, 2001.

[8] P. Dupuis and H. Ishii. On Lipschitz continuity of the solution mapping to the Skorokhod problem, with applications. Stochastics, 35:31-62, 1991.

[9] P. Dupuis and H. Ishii. On oblique derivative problems for fully nonlinear second-order elliptic PDE's on domains with corners. Hokkaido Math J., 20:135-164, 1991.

[10] P. Dupuis and K. Ramanan. Convex duality and the Skorokhod Problem, II. Prob. Th. and Rel. Fields, 115:197-236, 1999.

[11] P. Dupuis and K. Ramanan. An explicit formula for the solution of certain optimal control problems on domains with corners. Probab. Th. and Math. Stat., page to appear, 2001.

[12] R. J. Elliott and N. J. Kalton. The Existence of Value in Differential Games, volume 126 of Memoirs of the Amer. Math. Society. AMS, 1972.

[13] J.M. Harrison and M.I. Reiman. Reflected Brownian motion on an orthant. The Annals of Probab., 9:302-308, 1981.

[14] J. W. Helton and M. R. James. Extending $H^{\infty}$ Control to Nonlinear Systems: Control of Nonlinear Systems to Achieve Performance Objectives. SIAM, 1999.

[15] C. Maglaras. A methodology for dynamic control policy design for stochastic processing networks via fluid models. In Proceedings of the 
36th IEEE Conference on Decision and Control, New York, 1997. IEEE Publishers.

[16] S.P. Meyn. Sequencing and routing in multiclass queueing networks. part i: Feedback regulation. SIAM J. on Control and Optimization, page To appear, 2001.

[17] M. I. Reiman and R. J. Williams. A boundary property of semimartingale reflecting Brownian motions. Prob. Theor. Rel. Fields, 77:87-97, 1988.

[18] M.I. Reiman. Open queueing networks in heavy traffic. Math. of Oper. Research, 9:441-458, 1984.

[19] R.T. Rockafellar. Convex Analysis. Princeton University Press, Princeton, 1970.

[20] G. Weiss. On optimal draining of re-entrant fluid lines. In F. P. Kelley and R. J. Williams, editors, Stochastic Networks, volume 71 of IMA Volumes in Mathematics and Its Applications. Springer-Verlag, New York, 1995.

[21] G. Weiss. Optimal draining of re-entrant fluid lines: some solved examples. In F. P. Kelley, S. Zachary, and I. Ziedins, editors, Stochastic Networks, Theory and Applications, volume 4 of Lecture Note Series. Claredon Press, Oxford, 1996. 